\title{
Evaluation of oxidative stress in migraine patients with visual aura - the experience of an Rehabilitation Hospital
}

\author{
Adriana Bulboaca ${ }^{1,4}$, Gabriela Dogaru²,4, Mihai Blidaru1, Angelo Bulboaca ${ }^{3,4}$, Ioana Stanescu ${ }^{3,4}$
}

Corresponding author: Gabriela Dogaru, E-mail address: dogarugabrielaumf@gmail.com

1. Department of Pathophysiology, Iuliu Haţieganu University of Medicine and Pharmacy, Cluj-Napoca, Romania 2 -Department of PRM, Iuliu Haţieganu University of Medicine and Pharmacy Cluj-Napoca, Cluj-Napoca, Romania 3. Department of Neurology, Iuliu Haţieganu University of Medicine and Pharmacy, Cluj-Napoca, Romania

4- Rehabilitation Hospital, Cluj-Napoca, Romania

\section{Abstract}

Background: Although there are previous studies regarding the migraine pathophysiology, the clinical entity of migraine with aura can have an different pathophysiological mechanism compared with migraine without aura. One of the most important mechanism in migraine is represented by increasing of oxidative stress. The aim of this study was to study the levels of two oxidative stress molecules: nitric oxide (NO) and malondialdehyde (MDA) in migraine with visual aura compared with migraine without aura. Material and Method: a Control group (healthy volunteers) of 37 patients and 58 patient with migraine divided in Group 1 (migraine with visual aura) and Group 2 (migraine without aura) were taken in the study. All the patient were assessed regarding the age, body mass index, blood pressure, basal glycaemia, smoking/non-smoking status, C reactive protein and fibrinogen. Visual aura was assessed regarding transitive negative visual symptoms or positive visual symptoms. Oxidative status was assessed by measurements of the plasma levels of NO and MDA. Results; C reactive protein was significantly increased in both group 1 and 2 compared with control group, but with no statistical difference between group 1 and 2. Oxidative stress (NO and MDA) was significantly increased in both group 1 and 2 compared with control group. There was also found a statistical difference regarding NO and MDA levels between group 1 and 2. Conclusions; patients with visual aura have more intense oxidative stress that can contribute to cortical spreading depression mechanism involved in migraine pathophysiology. This difference between oxidative status in migraine with aura compared with migraine without aura can influence different clinical presentation of these patients. consequently, this difference can be a guide for further individualized therapies for migraine patients.

Key words: migraine, nitric oxide, malondialdehyde, migraine with visual aura, oxidative stress

\section{Introduction}

Migraine is an episodic headache disorder accompanied by various neurological, gastrointestinal and autonomic changes. In one fifth of the migraineurs, a neurological disturbance (visual, sensory or motor) appears during or before the development of the headache called migraine aura [1]. Aura symptoms of these different types usually follow one another in succession, beginning with visual, then sensory, then aphasic; but the reverse and other orders have been noted. The accepted duration for most aura symptoms is 1 hour, but motor symptoms are often longer lasting. Visual auras are the most common form of migraine aura [2]. It may consist of positive or negative visual symptoms and cortical spreading depression is felt to be the phenomenon that underlies it. Even in migraine without aura, vision it is not totally excluded given that one of the major criteria for the diagnosis of migraine is photophobia [3]. Visual symptoms associated with migraine (during and between attacks) consists in increased photophobia, increased symptoms of dry eyes, negative transient visual symptoms changes (scintillating scotoma, obscuration of the vision, tunnel vision, hemianopsia), and positive persistent visual phenomenon (visual snow, floaters or metamorphopsia with distorted contours and lightening symptoms) [4]. During the migraine attack the usually the visual symptoms are coming first (visual aura), followed by pain attack [5]. Its pathophysiology in not completely understood, but visual auras might be related to a self-propagating wave of cortical depolarization (cortical spreading depression), triggering the trigeminal-vascular system and leading to headaches [5]. Cortical spreading depression (CSD) is the underlying phenomenon in migraine with and without aura. In migraine without aura, CSD probably does not reach symptoms threshold. Normal vision is important in migraine, as lack of sight may change the visual experience during migraine aura, probably due to cortical reorganization and changes in local susceptibility to CSD [4] The migraine threshold of an individual depends on the balance between stimulation and inhibition in the central nervous system via oxidative phosphorylation in mitochondria [6-8]. Oxidative stress is an imbalance between the production of reactive oxygen species 
(ROS) and their elimination by antioxidants [9] One of the most important molecules in oxidative stress, involved in migraine patophysiology are nitric oxide and malondyaldehide (MDA) [10]. Contribution of nitric oxide (NO) to the migraine pathophysiology is still under research [11,12]. Malondialdehyde (MDA) is another major indicator of oxidative stress and is widely used as an index of lipid peroxidation. Plasma MDA levels are significantly elevated in migraine patients compared to healthy controls [13] Oxidative stress can induce DNA damage and changes in or variations of DNA repair genes that have been associated with the development of migraine in various clinical studies [14,15].

The aim of this study was to assess the oxidative stress (by NO and MDA assessment) in migraine patients with visual aura, and in patients with migraine and non-visual aura, to compare this parameters in this to groups and to observe an eventual correlation with inflammatory parameters as are $\mathrm{C}$ reactive and fibrinogen.

\section{Material and method}

This study was made on 58 patients with migraine (episodic attacks) diagnosis admitted into the Rehabilitation Hospital, Cluj-Napoca, Romania, Neurology and Balneology Departments, during a 3 years period. Patients signed an informed consent approved by the Ethics Committee of Clinical Rehabilitation Hospital. This group was subsequently divided in Group 1 - patients with migraine and visual aura (39), and Group 2 - patients with migraine without visual aura (19 patients). 37 patients were included in the Control group (healthy volunteers). The patients were questioned about visual symptoms during migraine attacks and the negative or positive symptoms were noted in 2 categories as follows: negative transitive symptoms (obscuration or total vision loss, scintillating scotoma, tunnel vision, hemianopsia), positive transitive symptoms (photophobia, visual snow, floaters, metamorphopsia with distorted contours, lightening in the visual field). An ophthalmologic examination was made and consisted in visual acuity assessment (best corrected visual acuity by LogMAR chart), contrast sensitivity test (Pelly-Robson chart), ophtalmoscopic examination and visual field assessment by computerized perimetry (Optopol). Patients with ophthalmologic diseases as are lens opacities, age related macular degeneration, diabetic retinopathy, optic neuropathy were excluded by ophthalmologic examination. Patients with stroke history (hemorrhagic/ischemic) were also excluded by neurologic examination. Comorbidities associated with migraine were also assessed: obesity assessed by body mass index (BMI), dysplipidemias (assessed by LDL-cholesterol, HDL-cholesterol, triglycerides), inflammatory parameters ( $\mathrm{C}$ reactive protein and fibrinogen), smoking, alcohol consumption and hypertension. Nitro-oxidative stress leading to endothelial dysfunction was assessed as nitric oxide synthesis expressed as NOx [16,17]. MDA was assessed according with the method described by Yagl [18]. The blood samples (fasting blood) were collected in a sterile EDTA vaccutainer at the least 72 hours after attack, centrifuged for plasma separation and kept at low temperature until further use.

Statistical analysis

The comparisons between groups for parametric data were made with Man-Whitney test for non-normal distributed data and with Chi-square test for nonparametric data. The values for parametric data were expressed as mean \pm standard deviations. p values less than 0.05 were considered significant. Spearman test was applied for correlation calculation of nonnormal distributed data.

\section{Results}

Demographics and clinical variables of the patients represented by age, body mass index (BMI), systolic blood pressure (SBP), diastolic blood pressure (DBP), smoking status (S), C reactive protein (CRP) and fibrinogen level are shown in table 1.

A statistical significant difference between groups was found for CRP value $(\mathrm{p}<0.01)$ (for both group 1 compared with control group and group 2 compared with control group). No other parameters were significant different between the groups. Smoking is also a statistical significant status when both Group 1 and Group 2 compared with control group, but with no difference between group 1 and 2 .

The best corrected visual acuity was between 0.9 an 1 , none of the patients presented pathological changes of contrast sensitivity and visual field assessment. Distribution of visual symptoms in the group of the patients with visual aura - in group 1 (34 patients): negative transitive symptoms (obscuration or total vision loss, scintillating scotoma, tunnel vision, hemianopsia), positive transitive symptoms (photophobia, visual snow or floaters, metamorphopsia with distorted contours, lightening in the visual field). 
Table 1 - Demographics and clinical variables

\begin{tabular}{|l|l|l|l|l|}
\hline Parameter & Control Group & Group 1 & Group 2 & p value (Group 1 vs Group 2) \\
\hline Age (years) & $38.5 \pm 3.33$ & $38.76 \pm 2.24$ & $37.23 \pm 2.89$ & NS \\
\hline BMI (Quetelet index) & $23.72 \pm 1.90$ & $25,75 \pm 2.01$ & $26,01 \pm 1.89$ & NS \\
\hline SBP (mmHg) & $115 \pm 12,33$ & $121.20 \pm 10.13$ & $118.66 \pm 8.89$ & NS \\
\hline DBP (mmHg) & $75.5 \pm 2.45$ & $76.66 \pm 5.56$ & $73.21 \pm 3.45$ & NS \\
\hline Basal glycemia & $90 \pm 2.33$ & $96.66 \pm 4.45$ & $91.33 \pm 3.47$ & NS \\
\hline S (active smoker/non-smoker) & $10 / 25$ & $30 / 9^{*}$ & $13 / 6^{*}$ & NS \\
\hline CRP (mg\%) & $0.47 \pm 0.18$ & $1.56 \pm 0.26^{*}$ & $1.21 \pm 0.23^{*}$ & p $<0.01$ \\
\hline Fibrinogen (mg\%) & $200.45 \pm 14.33$ & $261.76 \pm 12.17$ & $256.34 \pm 16.34$ & NS \\
\hline
\end{tabular}

${ }^{*} \mathrm{p}<0.01$ compared with control group, $\mathrm{NS}=$ non-significant

Visual symptoms, distributed as negative or positive symptoms are represented in table 2.

Table 2 - Distribution of visual symptoms in the group of the patients with visual aura

\begin{tabular}{|l|l|}
\hline Negative transitive symptoms & Total = $34(58 \%)$ \\
\hline - obscuration or total vision loss & 14 \\
\hline - scintillating scotoma & 7 \\
\hline - tunnel vision & 1 \\
\hline - hemianopsia & 2 \\
\hline Positive transitive symptoms & Total = 15 (42 \%) \\
\hline - photophobia & 7 \\
\hline - visual snow or floaters & 5 \\
\hline $\begin{array}{l}\text { - metamorphopsia with distorted } \\
\text { contours }\end{array}$ & 1 \\
\hline - lightening in the visual field & 3 \\
\hline
\end{tabular}

Negative visual symptoms were more frequent compared with positive visual symptoms in patients with migraine with aura. The obscuration or total vision loss were the most frequent symptoms, in the group of the patients with negative visual symptoms. Visual snow and floaters were the most frequent positive symptoms.

Oxidative status represented by NO and MDA plasma level is shown in table 3.

Table 3. Oxidative stress parameters: NO and MDA in group 1 and 2.

\begin{tabular}{|l|l|l|l|l|}
\hline Parameter & Control & Group 1 & Group 2 & $\begin{array}{l}\mathrm{p} \text { value } \\
\text { (Group 1 } \\
\text { versus } \\
\text { Group 2) }\end{array}$ \\
\hline $\begin{array}{l}\mathrm{NO} \\
(\mu \mathrm{mol} / \mathrm{L})\end{array}$ & $\begin{array}{l}28.48 \\
\pm 3.118\end{array}$ & $\begin{array}{l}34.655 \\
2.438^{*}\end{array}$ & $\begin{array}{l}30.263 \\
3.088^{*}\end{array}$ & $\mathrm{p}<0.00001$ \\
\hline $\mathrm{MDA}$ & $2.593 \pm$ & $5.911 \pm 0.2$ & $\begin{array}{l}3.4 \\
0.385^{*}\end{array}$ & $\mathrm{p}<0.00001$ \\
$(\mathrm{nmol} / \mathrm{L})$ & 0.239 & $15^{*}$ & 0.001 \\
\hline
\end{tabular}

$* \mathrm{p}<0.00001$ compared with control group

There were a significant differences when compared both group 1 and 2 with control group. The differences between study groups were significant for both oxidative stress parameters (NO and MDA) with $\mathrm{p}<0.0001$ (group 1 versus group 2 )

\section{Discussions}

Inflammatory mechanism can be involved in pathogenesis of migraine patients, showed by our study due to the increased CRP in both study groups compared with control group. Other studies also demonstrated the contribution of inflammatory mechanism in migraine pathophysiology, neurogenic inflammation being one of the most important mechanism involved in this disease [19,20]. Smoking was also an important contributing factor to migraine pathophysiology as our result demonstrated, being demonstrated as an factor which enhance oxidative stress [21]. Inflammation and oxidative stress are closely associated events, inflammation being considered as one of the consequences of oxidative stress, as the pathways that investigate the inflammatory mediators are triggered by oxidative stress molecules [22]. The correlation between oxidative stress and pathophysiology of migraine has been proved by previous studies, nitric oxide being one of the most studied molecule due to its involvement in the regulation of cerebral and extra cerebral cranial blood flow and arterial diameters [2325] . Increased endothelial nitric oxide synthase (eNOS) activity in the endothelium causes NO production and smooth muscle relaxation by direct activation of the NO-cGMP pathway, and may be involved in the initial headache response [26]. Based on the accumulating evidence that endogenous NO regulation is altered in migraine pathogenesis, global and isoform-selective inhibitors of NOS have been targeted for migraine drug development [25]. It is also involved in nociceptive processing by inducing cephalic hyperalgesia that arises as a consequence of central sensitization in pain pathways that sub serve to meningeal nociception [27].Combination of natural compounds as is Curcumin can improve the nociception modulation and decrease the NO level 
when added to Sumatriptan treatment in experimental migraine [17].

Migraine is a risk factor for the formation of silent brain white matter lesions (WMLs) that are possibly ischemic in nature. Although dysfunction of the Larginine/nitric oxide (NO) pathway has been associated with oxidative stress and endothelial dysfunction in migraine, its role in WML development has not been specifically investigated. Elevated L-arginine serum levels (an NO substrate) might reflect an increased demand for NO synthesis [28]. WMLs are also correlated with cognitive decline and nitro-oxidative stress [29].

Nitric oxide (NO) is involved in the pathogenesis of migraine pain and is formed after cortical spreading depression, the level in migraine patients with aura being significantly higher than in those without aura. Therefore preventive therapy with NO syntase inhibitors added to inhibitor and 5HT-1B/1D receptor agonist can reduce the frequency of crises [30]. Unfortunately, this NOS inhibitor raises cardiovascular safety concerns and has an unfavourable pharmacokinetic profile. As experimental studies predicted, iNOS inhibitors are ineffective in migraine. Still, upcoming selective nNOS inhibitors are under the study for migraine treatment, nNOS isoform being most clearly involved in trigeminovascular transmission and central sensitization [31].

Comparing the level of MDA in migraine patients with tension type headache patients, Gupta at al found that migraine patients had higher values of MDA than the subjects in the other group $(\mathrm{P}<0.001)$. $\mathrm{T}$ This study suggests that oxidative stress continues even between headache episodes in migraine patients but not in those with tension type headache patients. This could probably be consequent to the different pathophysiological mechanisms of migraine compared with tension type headache [32]. All the cranial autonomic symptoms were found to be more common in migraineurs as compared to tension type subjects (conjunctival injection, hyperlacrimation, periorbital swelling, and nasal symptoms [33]. Tuncel at al also found that the MDA levels of patients in the migraine group compared with control group were significantly higher. They found no significant correlation between MDA level and headache attack period. This study, had found increased oxidative stress (MDA and superoxide dismutase) in the patients with migraine with aura compared with migraine without aura [34].

It is suggested that the migraine generator network (MGN) is driven and may therefore respond differently to different spatio-temporal noxious input in the migraine subtypes migraine with aura (MWA) and migraine without aura (MWoA.) The noxious input is caused by a cortical perturbation of homeostasis, known as spreading depression (SD). The MGN might even trigger spreading depression mechanism in the first place by a failure in vasomotor control, as a consequence of endothelial dysfunction [35]. As a result, migraine could be considered as an dynamical disease in which endothelial dysfunction can lead to increasing of noxious stimuli input and enhancement of cortical spreading depression. In our study the increased oxidative stress and endothelial dysfunction found both group can contribute to migraine pathophysiology due to triggering the cortical spreading depression. By fMRI study was demonstrated that interictal brain activity is more intense in patients with migraine with aura compare with patients with migraine without aura that demonstrated that there is and increased brain hyperexcitability in patients with migraine with aura [36]. Datta et al found that despite similar interictal symptoms of visual discomfort, only migraine with aura subjects have cortical hyperresponsiveness to visual stimulus, suggesting a direct connection between cortical hyperresponsiveness and aura itself [37]. The responses were also greater in the lateral geniculate nuclei in migraine with aura, with no difference between migraine and control groups. Whole brain analysis showed that increased activation in MWA was confined to the occipital lobe [37]. Increased interictal visual sensitivity is present both in migraine with aura and migraine without aura patients, but significantly higher in migraine with aura patients [38].

\section{Conclusions}

Further knowledge about this issue may contribute the cause and complications of migraine and may be essential for development of treatment approaches. The difference of oxidative stress between the patients with aura compared with the patient without aura can represent an important factor for administration of an individual therapy related to patient's symptoms. This can be an important starting point in a personalized therapy approach in migraine patients. 


\section{Bibliography}

1. Alemdar $M$, Selekler $M$, Migraine and cortical spreading depression, Agri, 2006;18(4):24-30.

2. The International Classification of Headache Disorders, 3rd edition (beta version), Cephalalgia. 2013; 33(9):629-808.

3. Marzoli SB, Criscuoli A,The role of visual system in migraine, Neurol Sci, 2017 May;38(Suppl 1):99-102. doi: 10.1007/s10072-017-2890-0.

4. Vincent MB, Vision and migraine, Headache, 2015 ; 55(4):595-9.

5. Bidot S, Biotti D, Migraine with visual aura,J Fr Ophtalmol, 2016 ; 39(6):554-9.

6. Alp R , Selek S, Alp SI, Taşkin A, Koçyiğit A,Oxidative and antioxidative balance in patients of migraine, Eur Rev Med Pharmacol Sci, 2010 ;14(10):877-82.

7. Ferroni P, Barbanti P, Della-Morte D, Palmirotta R, Jirillo E, Guadagni F. Redox mechanisms in migraine: novel therapeutics and dietary interventions, Antioxid Redox Signal, 2017; Epub. [PubMed]

8. Erol I, Alehan F, Aldemir D, Ogus E, Increased vulnerability to oxidative stress in pediatric migraine patients. Pediatr Neurol. 2010;43(1):21-24.

9. Eren Y, Dirik E, Neşelioğlu S, Erel Ö, Oxidative stress and decreased thiol level in patients with migraine: cross-sectional study, Acta Neurol Belg, 2015; 115(4):643-9.

10. Mehmet Yigit, Ozgur Sogut, Özlem Tataroglu, Adnan Yamanoglu, Eda Yigit, Eray Metin Güler, Omer Faruk Ozer, and Abdurrahim Kocyigit Oxidative/antioxidative status, lymphocyte DNA damage, and urotensin-2 receptor level in patients with migraine attacks, Neuropsychiatr Dis Treat. 2018; 14: 367-374.

11. Neri M, Frustaci A, Milic M, et al. A meta-analysis of biomarkers related to oxidative stress and nitric oxide pathway in migraine. Cephalalgia. 2015;35(10):931-937.

12. Bulboacă AE, Bolboacă SD, Stănescu IC, Sfrângeu CA, Bulboacă AC, Preemptive Analgesic and Antioxidative Effect of Curcumin for Experimental Migraine, Biomed Res Int, 2017:4754701. doi: 10.1155/2017/4754701. Epub 2017 Oct 24.

13. Aytaç B, Coşkun Ö, Alioğlu B, Decreased antioxidant status in migraine patients with brain white matter hyperintensities, Neurol Sci, 2014;35(12):1925-1929.

14. Geyik S, Altunısık E, Neyal AM, Taysi S, Oxidative stress and DNA damage in patients with migraine, $\mathrm{J}$ Headache Pain. 2016;17:10. doi: 10.1186/s10194016-0606-0. Epub 2016 Feb 17.
15. Cetinkaya Y, Dasdemir S, Gencer M, DNA repair gene variants in migraine. Genet Test Mol Biomarkers. 2014;18(8):568-573

16. Bulboaca, AE; Ursu, C; Uifalean, A; Bulboaca AC, Correlation between migraine severity and comorbidities in episodic migraine patients, Romanian Journal of Neurology, 2015, Vol. 14 Issue 2, 85-89

17. Bulboacă AE, Bolboacă SD, Stănescu IC, Sfrângeu CA, Porfire A, Tefas L, Bulboacă AC, The effect of intravenous administration of liposomal curcumin in addition to sumatriptan treatment in an experimental migraine model in rats, Int J Nanomedicine. 2018 ;13:3093-3103. doi: 10.2147/IJN.S162087

18. Yagl K, Assay for blood plasma and serum peroxides, Methods Enzymol. 1984;105:28-31.

19. Su M, Ran Y, He Z , Zhang M, Hu G, Tang W , Zhao $\mathrm{D}, \mathrm{Yu} \mathrm{S}$, Inhibition of toll-like receptor 4 alleviates hyperalgesia induced by acute dural inflammation in experimental migraine, Mol Pain. 2018 ;14:1744806918754612. doi: $10.1177 / 1744806918754612$

20. Malhotra R, Understanding migraine: Potential role of neurogenic inflammation. Ann Indian Acad Neurol, 2016 ;19(2):175-82. doi: 10.4103/09722327.182302.

21. Rahman I, MacNee W. Oxidative stress and regulation of glutathione in lung inflammation. Eur Respir J. 2000;16(3):534-54.

22. Haddad JJ. Oxygen-sensing mechanisms and the regulation of redox-responsive transcription factors in development and pathophysiology, Respir Res, 2002;3:26.

23. Olesen J, The role of nitric oxide (NO) in migraine, tension-type headache and cluster headache, Pharmacol Ther, 2008;120(2):157-71. doi: 10.1016/j.pharmthera.

24. Fujioka K, Oishi M, Fujioka A, Nakayama $T$, Increased nitroglycerin-mediated vasodilation in migraineurs without aura in the interictal period, J Med Ultrason (2001), 2018 , doi: 10.1007/s10396018-0880-3. [Epub ahead of print]

25. Pradhan AA, Bertels Z, Akerman S, Targeted Nitric Oxide Synthase Inhibitors for Migraine, Neurotherapeutics, 2018 ;15(2):391-401. doi: 10.1007/s13311-018-0614-7.

26. Akerman S, Williamson DJ, Kaube H, Goadsby PJ, Nitric oxide synthase inhibitors can antagonize neurogenic and calcitonin gene-related peptide induced dilation of dural meningeal vessels, $\mathrm{Br} \mathrm{J}$ Pharmacol. 2002 ;137(1):62-8.

27. Flores Ramos JM, Devoize L, Descheemaeker A, Molat JL, Luccarini P, Dallel R. The nitric oxide donor, isosorbide dinitrate, induces a cephalic cutaneous hypersensitivity, associated with 
sensitization of the medullary dorsal horn. Neuroscience, 2017, ;344:157-166. doi: 10.1016/j.neuroscience.2016.12.028.

28. Erdélyi-Bótor S, Komáromy H, Kamson DO, Kovács N, Perlaki G, Orsi G, Molnár T, Illes Z, Nagy L, Kéki S, Deli G, Bosnyák E, Trauninger A, Pfund Z. Serum L-arginine and dimethylarginine levels in migraine patients with brain white matter lesions, Cephalalgia, 2017 ;37(6):571-580. doi: $10.1177 / 0333102416651454$.

29. Bulboacă AE, Bolboacă SD, Bulboacă AC, Prodan $\mathrm{CI}$, Association between low thyroid-stimulating hormone, posterior cortical atrophy and nitrooxidative stress in elderly patients with cognitive dysfunction, Arch Med Sci, 2017 ;13(5):1160-1167. doi: 10.5114/aoms.2016.60129

30. Hougaard A, Hauge AW, Guo S, Tfelt-Hansen P, The nitric oxide synthase inhibitor and serotonin-receptor agonist NXN-188 during the aura phase of migraine with aura: A randomized, double-blind, placebocontrolled cross-over study, Scand J Pain, 2013 ;4(1):48-52. doi: 10.1016/j.sjpain.2012.08.002.

31. Barbanti P, Egeo G, Aurilia C, Fofi L, Della-Morte $\mathrm{D}$, Drugs targeting nitric oxide synthase for migraine treatment, Expert Opin Investig Drugs, 2014 ; 23(8):1141-8. doi: 10.1517/13543784.2014.918953.

32. Gupta R, Pathak R, Bhatia MS, Banerjee BD. Ann Indian Acad Neurol. Comparison of oxidative stress among migraineurs, tension-type headache subjects, and a control group. 2009 ;12(3):167-72. doi: 10.4103/0972-2327.56316.

33. Gupta R, Bhatia MS, Comparison of clinical characteristics of migraine and tension type headache, Indian J Psychiatry, 2011;53(2):134-9. doi: 10.4103/0019-5545.82538.

34. Tuncel D, Tolun FI, Gokce M, Imrek S, Ekerbiçer H. Oxidative stress in migraine with and without aura, Biol Trace Elem Res, 2008 ;126(1-3):92-7. doi: 10.1007/s12011-008-8193-9.

35. Dahlem MA,Migraine generator network and spreading depression dynamics as neuromodulation targets in episodic migraine, Chaos, 2013;23(4):046101. doi: 10.1063/1.4813815.

36. Faragó P, Tuka B,Tóth E, Szabó N, Király A, Csete G, Szok D, Tajti J, Párdutz Á, Vécsei L, Kincses ZT. Interictal brain activity differs in migraine with and without aura: resting state fMRI study. J Headache Pain, 2017 ;18(1):8. doi: 10.1186/s10194-016-07168.

37. Datta R1, Aguirre GK, Hu S, Detre JA, Cucchiara B, Interictal cortical hyperresponsiveness in migraine is directly related to the presence of aura. Cephalalgia, 2013;33(6):365-74.

doi: 10.1177/0333102412474503.

38. Cucchiara B, Datta R, Aguirre GK , Idoko KE , Detre $\mathrm{J}$, Measurement of visual sensitivity in migraine: Validation of two scales and correlation with visual cortex activation, Cephalalgia, 2015;35(7):585-92. doi: $10.1177 / 0333102414547782$. 